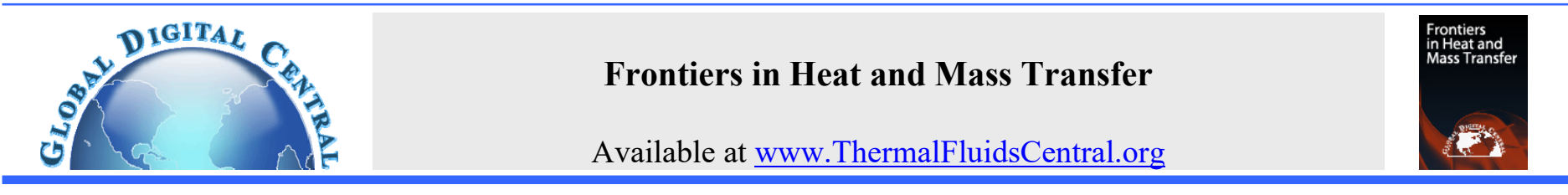

\title{
ALGORITHM AND INFLUENCE FACTOR STUDY ON FLOW PATTERN TRANSITION FROM STRATIFIED FLOW TO NON-STRATIFIED FLOW OF GAS-LIQUID TWO-PHASE FLOW
}

\author{
Rongge Xiao ${ }^{\mathrm{a} .}{ }^{*}$, Dong Wang ${ }^{\mathrm{a}}$, Shuaishuai Jin ${ }^{\mathrm{a}}$, Hongping Yu ${ }^{\mathrm{b}}, \mathrm{Bo} \mathrm{Liu}^{\mathrm{a}}$ \\ a Shaanxi Key Laboratory of Advanced Stimulation Technology for Oil \& Gas Reservoirs, College of Petroleum Engineering, Xi'an Shiyou \\ University, Xian 710065, Shaanxi China, \\ ${ }^{b}$ Production Technology Research Institute of The Second Oil Production Plant of Changqing Oilfield. Qingyang 745100, Gansu, China
}

\begin{abstract}
Based on the Viscous Kelvin-Helmholtz theory used by D. Barnea \& Y. Taitel (1993), a two-fluid stratified flow model of gas-liquid two-phase flow is established. Using the mathematical derivation, the influence of various influence factors on the stability of liquid level structure is synthesized. Compared with the criteria of D Barnea \& Y. Taitel (1993) and Taitel \& Dukler(1976), and the algorithm of flow pattern transition criterion of stratified flow is proposed. According to the data of multiphase flow experimental loop, the influence of liquid viscosity and the instantaneous volume flow rate change of gas-liquid two-phase on the transition criterion is analyzed. The result shows that liquid viscosity has a great influence on the stability of stratified flow. With the increase of liquid viscosity, the friction resistance and viscous force increase, and they play a role in preventing the rise of liquid level height, it is difficult to form liquid slug in the pipe, so that the region where the stratified flow exists stably is enlarged. And instantaneous change of gas-liquid flow rate has little influence on the flow pattern transition.
\end{abstract}

Key words: Stratified flow; Two-fluid model; Flow pattern transition; gas-liquid two-phase flow

\section{INTRODUCTION}

For the gas-liquid two-phase flow pattern in the pipeline, domestic and foreign scholars have carried out a lot of experimental and theoretical studies. Models for predicting flow pattern transitions during steady gas-liquid flow in vertical tubes were developed by Taitel Y based on physical mechanisms suggested for each transition. These models incorporate the effect of fluid properties and pipe size and thus are largely free of the limitations of empirically based transition maps or correlations. Experimental and analytical studies were presented directed to the prediction of flow pattern transitions in a vertical rod bundle array. Based on physical interpretation of the transition mechanisms, analytical models were given by Venkateswararao P. The experimental measurements related to the stratified-slug flow transition in a horizontal pipe containing a 7-rod bundle were described by Krishnan V S. The results were compared with the predictions of a modified form of the Taitel-Dukler theory. The predictions were also compared with other published experimental data on the stratified-slug flow transition in air-water and steam-water flows in pipes containing 37-rod bundles. In 2000, a unified steady-state two-phase flow mechanistic model for the prediction of flow pattern, liquid holdup and pressure drop was presented that is applicable to the range of inclination angles from horizontal $\left(0^{\circ}\right)$ to upward vertical flow $\left(90^{\circ}\right)$. The proposed model implemented new criteria for eliminating discontinuity problems and provided smooth transitions between the different flow patterns. A theoretical study was conducted to investigate flow-pattern transitions in slightly inclined pipelines carrying oil/water mixtures. Two criteria obtained in the linear-stability theory of stratified oil/water flow were used to predict the transition from a stratified to non-stratified flow pattern by Yuri V. The effects of liquid properties on flow pattern transition and pressure drop during gas-liquid flow were experimentally investigated by Jingyu X. It was shown that the properties of the liquid exert a moderate effect on the locations of the transition lines from plug to slug flow in horizontal and from annular-mist to intermittent flow in vertical downward, but undertake a minimal effect on transition from stratified wary to intermittent flow in horizontal and flow pattern transition in vertical upward. The transition mechanisms between the different flow patterns were analyzed and the corresponding transitional models were proposed by Hu Zhihua. And local flow pattern maps of the upward gas-water two-phase flow in the vertical tube with a horizontal rod were constructed. Based on the slug stability model and simplified stratified flow model, the predictions of the critical liquid heights and critical superficial velocities for the transition of a stratified flow to a slug flow in a horizontal $2.54 \mathrm{~cm}$ pipe was provided by Liu Y $P$. And the influence of the variation in gas-liquid interfacial friction factor on the prediction of flow regime transition was also analyzed. Theoretical relations that predict the transition from a stratified pattern to a slug pattern were presented by Liu Yiping, including a onedimensional wave model that contains less empiricism than the commonly used Taitel-Dukler model, and the ideal model for stratified flow for the gas-liquid flow in horizontal pipes. And theoretical analysis was conducted on the stratified to slug flow regime transition. The friction, existing between the fluid and pipe wall, and on the interface of two phases, was especially taken into account. The effect of pipe diameter on the transition was also studied. Zhang JinHong put forward a transition criterion in rolling state where the dispersed bubble flow was transformed into bubble flow or intermittent flow; or the annular flow was changed into intermittent flow or intermittent-plus-stratified flow. The determination of the flow regimes is primarily based on flow regime maps or transition criteria, which are developed for steady-state, fully-developed flows. It is of importance to model the flow regime transition dynamically for more accurate predictions of two-phase flows.

\footnotetext{
* Corresponding Author. E-mail: xiaorongge@163.com.
} 
Therefore, Wang Xia developed a dynamic modeling strategy for determining flow regimes in gas-liquid two-phase flows through the introduction of interfacial area transport equations (IATEs) within the framework of a two-fluid model. The gravity differential pressure fluctuation signal of Gas-liquid two-phase flow in horizontal pipeline was researched by De Fang L, it directly related to phase holdup of two-phase flow, and could reflect the variation of interphases force. The characteristic parameters of differential pressure signals were extracted by wave analysis, which was sensitive to the conversion from stratified flow to annular flow, so it proved a new way to flow pattern identification for gas-liquid two-phase flow and provided a strong evidence to reveal the flow pattern transition mechanism of gas-liquid two phase flow. Based on Kelvin-Helmholtz stability criteria for stratified flow in literature, the new criteria of transition from stratified flow to non-stratified flow for gas-liquid flow in horizontal wellbore was developed by Xiao R, in which the influence of wall inflow was considered. Experimental researches and prediction calculations were also done in order to analyze how much wall inflow affected transition conditions. Bhagwat S M developed an empirical model to predict the existence of stratified flow in horizontal and downward inclined gasliquid two-phase flow. The proposed model is in non-dimensional form, and the empirical parameters used in the proposed model accounted for the effect of pipe diameter, pipe orientation, and the liquid density on the transition line between stratified and non-stratified flow. In order to improve the high-viscosity oil flow-pattern transition modeling in horizontal pipes for the stratified-smooth (SS)/stratified-wavy (SW) and intermittent/annular transitions. Two transitions were improved by introducing a liquid viscosity-dependent sheltering coefficient model and a new high-viscosity liquid-level criterion by Eissa Al-Safran. The existing inviscid Kelvin-Helmholtz (IKH), viscous Kelvin-Helmholtz (VKH), and Taitel and Dukler models for the stratified/non-stratified (S-NS) transition were also investigated. Fedor Ronshin had developed a new technique that makes it possible to measure the local void fraction, size of characteristic regions of the liquid films on the upper and lower walls of micro-channel, frequency of bubble formation, and other quantitative characteristics. Based on that developed methodology, criteria that accurately determine the boundaries between the two-phase flow regimes were created. The features of a two-phase flow in a microchannel were determined and a new flow regime map were created.

However, most of these transition criteria of gas liquid two phase flow are based on the basic hydraulic model of two-phase flow. Since the judgment of flow pattern is different from the hydraulic calculation, it is not necessary to strictly consider the influence of various flow factors. In order to facilitate the analysis and derivation, the model should be simplified first. General assumption: gas and liquid flow are isothermal in the pipe section, and the heat transfer between the fluid and the outside world can be ignored; the mass transfer and momentum transfer between the gas and liquid phases in the pipe section are very small, and it has no obvious influence on the flow pattern transition.

Furthermore, previous studies of flow pattern transition of stratified flow does not take into account the instantaneous flow rate change along the pipeline.(In recent years, natural gas, as a clean energy, plays a more and more important role in the national economy, and in the wet gas and condensate gas pipelines where oil and gas are mixed, the most common one is gas-liquid two-phase stratified flow, because of the change of external temperature and pressure, the instantaneous flow rate of gas-liquid stratified flow is constantly changing.) So it is not suitable to predict the instantaneous flow rate changes of gas liquid two phase flow of stratified flow. In horizontal wet gas wellbore (the changes of wellbore temperature and pressure are very dramatic) and in horizontal wet gas pipe, small instantaneous flow rate change will hinder the formation of liquid film at the wall of the tube when the stratified flow of gas-liquid two-phase flow is converting into non-stratified flow, and make the formation condition of flow pattern more stringent than the common flow pattern transition criteria of gas-liquid two-phase flow. Therefore, based on the theoretical basis, this study revises the flow pattern transition criteria of gas-liquid two- phase flow from stratified flow to non-stratified flow, and proposes a new transition criterion on the basis of previous research results.

\section{FORMATION MECHANISM OF STRATIFIED FLOW}

Stratified flow is the most common flow pattern in gas-liquid two-phase flow pipeline. Stratified flow is a separation flow pattern with relatively stable phase structure, its gas and liquid phases flow in the pipeline respectively, and there is no obvious wave generation between phases.

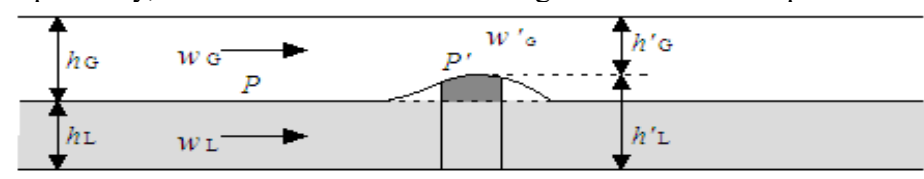

Fig. 1. Schematic of simplified Kelvin-Helmholtz stability analysis

In 1976, Taitel and Dukler used non-viscous Kelvin Helmholtz stability theory to analyze the structure between gas and liquid phase of stratified flow in horizontal and slightly tilted gas-liquid two-phase pipelines, as shown in figure 1, they ignored the influence of liquid viscosity on flow pattern and approximately believed that hydraulic friction coefficient between gas and liquid phase is equal to the hydraulic friction coefficient between the gas phase and pipe wall. And they assumed that the viscosity of liquid is much higher than gas viscosity, gas phase velocity is much larger than the liquid velocity of near the interface. Using the mathematical model of steady-state flow, they obtained the criteria for judging the stable existence of the interface of stratified flow, and the expression is:

$w_{G}>K_{T D}\left[\frac{g A_{G}\left(\rho_{L}-\rho_{G}\right) \cos \theta}{\rho_{G} \frac{d A_{L}}{d h_{L}}}\right]^{0.5}$

Where, the following equation is used to estimate $K_{T D}: K_{T D}=1-\frac{h_{L}}{d} ; \theta$ is the pipe inclination angle; if it is a horizontal pipe, then $\theta=0^{\circ}, \cos \theta=1$, the expression of $\mathrm{V}-\mathrm{H}$ criterion of stratified flow is:

$$
w_{G}>K_{T D}\left[\frac{g A_{G}\left(\rho_{L}-\rho_{G}\right)}{\rho_{G} \frac{d A_{L}}{d h_{L}}}\right]^{0.5}
$$

Although equation (1) can be used to determine the transition from stratified flow to other flow patterns, the solution of the height of liquid level can be obtained by using the momentum balance equation which includes viscosity factor. But as far as the criterion itself is concerned, it is derived from non-viscous theory, so it can only apply to low-viscous fluids or non-viscous fluids. Other scholars also pointed out that Taitel criteria has defects due to insufficient consideration of liquid phase viscosity.

\section{RESTABLISHMENT OF TRANSITION CRITERIA OF STRATIFIED FLOW TO NON-STRATIFIED FLOW}

Theoretical and experimental studies show that stratified flow occurs when the velocity of gas and liquid phase are relatively low, and the specific boundary of stratified flow depends on the physical properties of the fluid and the geometric size of the tube. With the increase of the velocity of liquid phase, waves are generated at the interface of gasliquid two phases, and the interface waves grow rapidly, so that the stratified flow converts to non-stratified flow. If the velocity of gas phase is high and there is not enough liquid phase in the pipe, even if 
the liquid phase which bridges the pipe wall has been formed, the liquid phase bridged in the pipe will be blown away and a thin liquid film will be formed on the pipe wall, and the annular flow will occur. With the increase of gas velocity, the liquid phase in the stratified flow is forced to flow to both sides of the pipe wall, and the interface becomes concave, while the share of the wet pipe wall becomes larger and larger, and the flow finally turns into the annular flow. Therefore, wet wall fraction 0.9 is commonly used in the Grolman relation to estimate the transition boundary in the transition criteria of the stratified flow to the annular flow. The method also ensures the continuous conversion of hydrodynamic calculation between stratified flow and annular flow. If the velocity of gas phase is low and the velocity of liquid phase gradually increases, it is enough to make the liquid phase completely bridge the pipe wall under the action of the interface wave, thus the liquid slug is forming and slug flow occurs, and the transition from stratified flow to slug flow occurs.

The fluid in gas-liquid two-phase flow pipeline is usually viscous fluid, and the influence of viscosity on fluid cannot be ignored. At the same time, for the wet natural gas pipeline, the conveying pressure is very high, and the density difference between the gas and liquid phases is not big. Therefore, in order to judge the gas-liquid flow pattern more accurately, starting from the basic mathematical model of stratified flow, considering the viscous effect of liquid phase, and making sure the derivation process is as rigorous as possible, Taitel-Dukler criterion is improved, and the stability criterion of stratified flow between phases with a wider range of application and a full reflection of various influence factors is obtained.

Based on the Viscous Kelvin-Helmholtz theory used by D. Barnea \& y. Taitel (1993), a two-fluid stratified flow model of gas liquid two phase flow is established. Figure 2 shows gas-liquid two-phase stratified flow diagram in horizontal pipeline. As shown in figure 2, gas phase and liquid phase flow is stratified, gas phase and liquid phase respectively flow at the upper and lower, droplet entrainment in the air can be ignored, and assume that each phase are one-dimensional flow, gas-liquid interface is smooth and flat, the interface friction coefficient is determined by gas wall friction coefficient. According to the two assumptions presented, and the static pressure gradient effect caused by the liquid level height in the pipe section is considered, by rewriting the equations of the hydraulic model, a mathematical model describing the stratified flow is obtained.
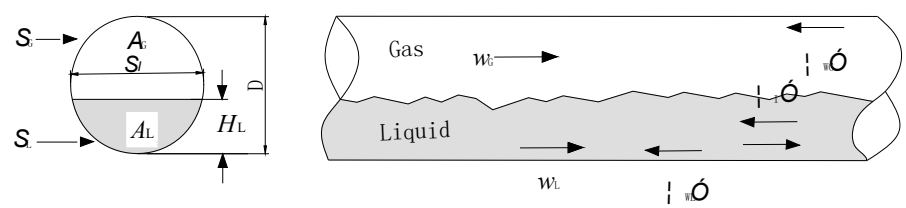

Fig. 2 Gas-liquid two-phase stratified flow diagram

\subsection{Continuity equation}

The gas phase:

$\frac{\partial}{\partial t}\left(\rho_{G} A_{G}\right)+\frac{\partial}{\partial x}\left(\rho_{G} A_{G} w_{G}\right)=\rho_{G} q_{I G}$

The liquid phase:

$\frac{\partial}{\partial t}\left(\rho_{L} A_{L}\right)+\frac{\partial}{\partial x}\left(\rho_{L} A_{L} w_{L}\right)=\rho_{L} q_{I L}$

Where, $q_{I G}$ and $q_{I L}$ are the volume flow rates of the gas phase and liquid phase in pipe per unit length respectively, $\mathrm{m}^{2} / \mathrm{s} ; \rho_{G}$ and $\rho_{L}$ respectively represent the density of gas phase and liquid phase, $\mathrm{kg} / \mathrm{m}^{3} ; A_{L}$ and $A_{G}$ are respectively the cross-sectional area occupied by liquid and gas phases in the pipeline, $\mathrm{m}^{2} ; w_{G}$ and $w_{L}$ respectively represent the true velocity of gas phase and liquid phase, $\mathrm{m} / \mathrm{s}$.

\subsection{The momentum equation}

The gas phase:

$\frac{\partial}{\partial t}\left(\rho_{G} A_{G} w_{G}\right)+\frac{\partial}{\partial x}\left(\rho_{G} A_{G} w_{G}^{2}\right)-A_{G} \frac{\partial P_{I G}}{\partial x}=-\tau_{w G} S_{G}-\tau_{I} S_{I}-\rho_{G} g A_{G} \cos \theta \frac{\partial h_{L D}}{\partial x}-\rho_{G} g A_{G} \sin \theta$
The liquid phase:

$\frac{\partial}{\partial t}\left(\rho_{L} A_{L} w_{L}\right)+\frac{\partial}{\partial x}\left(\rho_{L} A_{L} w_{L}^{2}\right)-A_{L} \frac{\partial P_{L L}}{\partial x}=-\tau_{w L} S_{L}+\tau_{I} S_{I}-\rho_{L} g A_{L} \cos \theta \frac{\partial h_{L D}}{\partial x}-\rho_{L} g A_{L} \sin \theta$

Where, calculation of dimensionless liquid holding height is $h_{L D}=\frac{h_{L}}{D}$, where $h_{L}$ is height of liquid phase, D is pipe diameter, $\mathrm{m}$; $\tau_{W G} 、 \tau_{W L}$ and $\tau_{I}$ is the shear stress between gas and wall、 liquid and wall 、 and between gas-liquid phases respectively, $\mathrm{Pa} ; P_{I G}$ and $P_{I L}$ respectively represent the pressure gradient of gas phase and liquid phase, $\mathrm{Pa} / \mathrm{m} ; S_{L} 、 S_{G}$ and $S_{I}$ are the length of liquid wetted perimeter、gas wetted perimeter and gas-liquid interface respectively, $\mathrm{m} ; \frac{\partial P}{\partial x}$ is the pressure gradient, $\mathrm{Pa} / \mathrm{m}$.

The approximate relation on the interface between the pressure of gas phase and liquid phase is:

$P_{I G}-P_{I L}=0$

Assuming that the fluid is incompressible, the flow area occupied by the gas-liquid phase is a function of the liquid level height in the pipe, so the continuity equation (3) and (4) are set up and expressed in the form of the liquid level height, and the following two equations can be derived:

$\frac{\partial h_{L D}}{\partial t}+\frac{A_{L}}{A_{L}} \frac{\partial w_{L}}{\partial x}+w_{L} \frac{\partial h_{L D}}{\partial x}=F_{I L}$
$\frac{\partial h_{L D}}{\partial t}-\frac{A_{G}}{A_{L}} \frac{\partial w_{G}}{\partial x}+w_{G} \frac{\partial h_{L D}}{\partial x}=F_{I G}$

Among them, $A^{\prime}{ }_{L}=\frac{d A_{L}}{d h_{L D}}$. The partial derivatives in equations

and (6) are expanded, and the continuity equations (3) and (4) of gas and liquid phases are substituted. According to equation (7), two momentum equations are set up to eliminate the pressure gradient, and the following equation can be obtained:

$$
\begin{aligned}
& \rho_{L} \frac{\partial w_{L}}{\partial t}-\rho_{G} \frac{\partial w_{G}}{\partial t}+\rho_{L} w_{L} \frac{\partial w_{L}}{\partial x}-\rho_{G} w_{G} \frac{\partial w_{G}}{\partial x}+\left(\rho_{L}-\rho_{G}\right) g \cos \theta \frac{\partial h_{L D}}{\partial x}=F \\
& \text { Where, } \\
& \left\{\begin{array}{c}
F=-\frac{\tau_{w L} S_{L}}{A_{L}}+\frac{\tau_{w G} S_{G}}{A_{G}}+\tau_{I} S_{I}\left(\frac{1}{A_{L}}+\frac{1}{A_{G}}\right)-\left(\rho_{L}-\rho_{G}\right) g \sin \theta-\left(\frac{\rho_{L} w_{L} q_{L}}{A_{L}}-\frac{\rho_{G} w_{G} q_{I G}}{A_{G}}\right) \\
F_{L}=\frac{q_{L}}{A_{L}} \\
F_{I G}=-\frac{q_{I G}}{A_{L}}
\end{array}\right.
\end{aligned}
$$

The equations $(8) \sim(10)$ are set up to eliminate the partial differential of $w_{L}, w_{G}$, and the single valued partial differential function of dimensionless liquid holding height $h_{L D}$ is finally simplified. In addition, dimensionless liquid level height can be expressed as the sum of its mean value and fluctuation value. Let $h_{L D}=\bar{h}_{L D}+\hat{h}_{L D}$, and,

$\left[\frac{\rho_{L} w_{L}^{2}}{H_{L}}+\frac{\rho_{G} w_{G}^{2}}{\varphi}-\left(\rho_{L}-\rho_{G}\right) g \cos \theta \frac{A}{A_{L}}\right] \frac{\partial^{2} \hat{h}_{L D}}{\partial x^{2}}$

$+2\left[\frac{\rho_{L} w_{L}}{H_{L}} \frac{\rho_{G} w_{G}}{\varphi}\right] \frac{\partial^{2} \hat{h}_{L D}}{\partial t \partial x}+\left[\frac{\rho_{L}}{H_{L}}+\frac{\rho_{G}}{\varphi}\right] \frac{\partial^{2} \hat{h}_{L D}}{\partial t^{2}}$

$=\left[-\frac{A}{A_{L}} \frac{\partial F}{\partial h_{L D}}-\frac{w_{G}}{\varphi} \frac{\partial F}{\partial w_{G}}+\frac{w_{L}}{H_{L}} \frac{\partial F}{\partial w_{L}}\right] \frac{\partial \hat{h}_{L D}}{\partial x}+\left[-\frac{1}{\varphi} \frac{\partial F}{\partial w_{G}}+\frac{1}{H_{L}} \frac{\partial F}{\partial w_{L}}\right] \frac{\partial \hat{h}_{L D}}{\partial t}$

Where, $H_{L}$ is the liquid holdup, that is, $H_{L}=\frac{A_{L}}{A}$. $\varphi_{\text {is }}$ the void fraction, its calculation formula is $\varphi=1-H_{L}=\frac{A_{G}}{A}$. The fluctuation value of dimensionless liquid level height can be expressed as: 


$$
\hat{h}_{L D}=\varepsilon e^{i(\omega t-k x)}
$$

Substituting (13) into (12) can we get:

$\omega^{2}-2(a k-b i) \omega+c k^{2}-e k i=0$

Where,

$a=\frac{1}{\rho}\left(\frac{\rho_{L} w_{L}}{H_{L}}+\frac{\rho_{G} w_{G}}{\varphi}\right)$

$b=\frac{1}{2 \rho}\left[\left(\frac{\partial F}{\partial w_{S L}}\right)_{w_{S G} E_{L}}-\left(\frac{\partial F}{\partial w_{S G}}\right)_{w_{S L} E_{L}}\right]$

$c=\frac{1}{\rho}\left(\frac{\rho_{L} w_{L}^{2}}{H_{L}}+\frac{\rho_{G} w_{G}^{2}}{\varphi}-\left(\rho_{L}-\rho_{G}\right) g \cos \theta \frac{A}{A_{L}}\right)$

$e=\frac{1}{\rho}\left[-\left(\frac{\partial F}{\partial H_{L}}\right)_{w_{S L} w_{S G}}-w_{G}\left(\frac{\partial F}{\partial w_{S G}}\right)_{w_{S L} E_{L}}+w_{L}\left(\frac{\partial F}{\partial w_{S L}}\right)_{w_{S G} E_{L}}\right]$

$\rho=\frac{\rho_{L}}{H_{L}}+\frac{\rho_{G}}{\varphi}$

From (14), we can get:

$\omega=(a k-b i) \pm \sqrt{\left(a^{2}-c\right) k^{2}-b^{2}+(e k-2 a b k) i}$

Let $\omega=r_{1}+r_{2} i$, according to equation (11), when the imaginary part $r_{2}<0$ of $\omega$, the amplification factor of the amplitude of the fluctuation value of dimensionless liquid holding height of the liquid phase $e^{-r_{2} t}>1$, as time goes on, the amplitude increases gradually and increases exponentially, that is, the liquid level height increases, the liquid slug occurs, and eventually, the gas-liquid interface is unstable, pipe sections become blocked and convert into other flow patterns. Therefore, the condition for the stable existence of the interface of gasliquid phase of stratified flow can be obtained as follows:

$r_{2} \geq 0$

Let $r_{2}=0$, since $\omega=r_{1}+r_{2} i$, substituting $r_{2}=0$ into equation (15) can we get:

$r_{1}=(a k-b i) \pm \sqrt{\left(a^{2}-c\right) k^{2}-b^{2}+(e k-2 a b k) i}$

Transfer left and right:

$r_{1}-a k+b i= \pm \sqrt{\left(a^{2}-c\right) k^{2}-b^{2}+(e k-2 a b k) i}$

Squaring the left and right sides at the same time, and get:

$\left(r_{1}-a k\right)^{2}-b^{2}+2 b\left(r_{1}-a k\right) i=\left(a^{2}-c\right) k^{2}-b^{2}+(e k-2 a b k) i$

The critical stability condition of the stratified flow is:

$\left(a k-r_{1}\right)^{2}-\left(a^{2}-c\right) k^{2}=0$

$2 r_{1} b-e k=0$

According to equation (18), $r_{1}=\frac{e k}{2 b}$, substitute it into equation

(17) can we get the critical condition of transition from stratified flow to non-stratified flow:

$\left(a-\frac{e}{2 b}\right)^{2}-\left(a^{2}-c\right)=0$

Substituting each item in equation (14) into equation (19), we can get:

$\left(C_{V}-C_{I V}\right)^{2}+\frac{\rho_{L} \rho_{G}}{\rho^{2} H_{L} \varphi}\left(w_{G}-w_{L}\right)^{2}-\frac{\left(\rho_{L}-\rho_{G}\right)}{\rho} g \cos \theta \frac{A}{A_{L}}=0$

where,

$$
\begin{gathered}
C_{V}=\frac{e}{2 b}=\frac{\left[-\left(\frac{\partial F}{\partial H_{L}}\right)_{w_{S L} w_{S G}}-w_{G}\left(\frac{\partial F}{\partial w_{S G}}\right)_{w_{S L} E_{L}}+w_{L}\left(\frac{\partial F}{\partial w_{S L}}\right)_{w_{S G} E_{L}}\right]}{\left[\left(\frac{\partial F}{\partial w_{S L}}\right)_{w_{S G} E_{L}}-\left(\frac{\partial F}{\partial w_{S G}}\right)_{w_{S L} E_{L}}\right]} \\
C_{I V}=a=\frac{\rho_{L} w_{L} \varphi+\rho_{G} w_{G} H_{L}}{\rho_{L} \varphi+\rho_{G} H_{L}}
\end{gathered}
$$

In equation (20), the second and third terms on the left side are the $\mathrm{K}-\mathrm{H}$ instability factors of the one-dimensional non-viscous ideal fluid between phases. The first term is the additional effect caused by shear stress, which amplifies any disturbance on the surface of the liquid film. $C_{V}$ is the propagation velocity of the interface unsteady wave, which is the main factor affecting the wave stability in the flow, and it reflects the effect of viscosity on flow pattern transition. For the flow of nonviscous fluid, since $e=b=0$, substituting it into equation (14) can we obtain the critical interface wave velocity $C_{I V}=a$ of non-viscous, that is, equation (14a)

After further deformation of Equation (20), a form similar to Taitel-Dukler criterion can be obtained. The condition for flow pattern transition from stratified flow to non-stratified flow is:

$\left|w_{G}-w_{L}\right|>K_{V}\left[\frac{\left(\rho_{L} \varphi+\rho_{G} H_{L}\right)}{\rho_{L}} \frac{\left(\rho_{L}-\rho_{G}\right) g \cos \theta}{\rho_{G}} \frac{A}{\frac{d A_{L}}{d h_{L}}}\right]^{0.5}$

Where,

$K_{V}=\left[1-\frac{\left(C_{V}-C_{V}\right)^{2}}{\frac{\left(\rho_{L}-\rho_{G}\right) g \cos \theta}{\rho} \frac{A}{\frac{d A_{L}}{d h_{L}}}}\right]^{0.5}$

When $\rho_{L}>>\rho_{G}, \frac{\rho_{L} \varphi+\rho_{G} H_{L}}{\rho_{L}} A \approx A_{G}$, then the transition condition (21) can be further simplified as:

$\left|w_{G}-w_{L}\right|>K_{V}\left[\frac{\left(\rho_{L}-\rho_{G}\right) g \cos \theta}{\rho_{G}} \frac{A_{G}}{d A_{L}} \frac{}{d h_{L}}\right]^{0.5}$

Stratified flow can also be subdivided into smooth stratified flow and wave stratified flow. Generally, the tiny waves generated in pipelines propagate downstream with the flow rate $\mathcal{C}$, and only when the gas flow rate $w_{g} \gg c$ can waves form, that is, wave stratified flow is formed from smooth stratified flow. According to this principle, the dimensionless criterion is derived as follows:

$K \geq \frac{2}{w_{G} \sqrt{S w_{L}}}$

where, $K=\left[\frac{\rho_{G} w_{S G}^{2} w_{S L}}{\left(\rho_{L}-\rho_{G}\right) v_{L} g \cos \theta}\right]^{0.5} ; v_{L}-$ - liquid kinematic viscosity;

S--Barrier factor. According to the experiment, Benjaming pointed out that the scope of $\mathrm{S}$ was $0.01-0.03$. In this study, $\mathrm{S}=0.018$ was used.

\section{COMPARISON BETWEEN TRANSITION CRITERIA FROM STRATIFIED FLOW TO NON- STRATIFIED FLOW AND PREVIOUS STUDY CRITERIA}

4.1 Comparison with D.Barnea \& Y.Taitel (1993) criterion Transition criteria formula (22) from stratified flow to non-stratified flow, that is, the flow pattern transition condition proposed in this study, 
looks exactly the same as transition condition from stratified flow to non-stratified flow proposed by D.Barnea \& Y.Taitel(1993), but the expressions of parameters $F$ are different. Therefore, the expressions of all the coefficients related to the parameter $F$, such as $K_{V} 、 C_{V}$ 、 $b$ and $e$, will also be different. In the transition model from stratified flow to non-stratified flow proposed in this study, if various influence factors such as viscosity and instantaneous flow rate change of gas and liquid are not taken into account and let the instantaneous flow rate change of each phase be equal to zero, it is similar to the transition model from stratified flow to non-stratified flow proposed by D.Barnea \& Y.Taitel, that is:

$$
\left|w_{G}-w_{L}\right|>K_{V}\left[\frac{\left(\rho_{L}-\rho_{G}\right) g \cos \theta}{\rho_{G}} \frac{A_{G}}{\frac{d A_{L}}{d h_{L}}}\right]^{0.5}
$$

Where,

$$
K_{V}=\left[1-\frac{\left(C_{V}-C_{I V}\right)^{2}}{\frac{\left(\rho_{L}-\rho_{G}\right) g-\sigma k^{2}}{\rho} \frac{A}{\frac{d A_{L}}{d h_{L}}}}\right]^{0.5}
$$

$C_{V}=\frac{e}{2 b}=\frac{\left(\frac{\partial F}{\partial H_{L}}\right)_{U_{S L} U_{S G}}}{\left[\left(\frac{\partial F}{\partial w_{S L}}\right)_{w_{S G} E_{L}}-\left(\frac{\partial F}{\partial w_{S G}}\right)_{w_{S L} E_{L}}\right]}$

$b=\frac{1}{2 \rho}\left[\left(\frac{\partial F}{\partial w_{S L}}\right)_{w_{S G} E_{L}}-\left(\frac{\partial F}{\partial w_{S G}}\right)_{w_{S L} E_{L}}\right]$

$e=-\frac{1}{\rho}\left(\frac{\partial F}{\partial H_{L}}\right)_{w_{S L} w_{S G}}$

$F=-\frac{\tau_{w L} S_{L}}{A_{L}}+\frac{\tau_{w G} S_{G}}{A_{G}}+\tau_{I} S_{I}\left(\frac{1}{A_{L}}+\frac{1}{A_{G}}\right)-\left(\rho_{L}-\rho_{G}\right) g \sin \theta$

Where, $\sigma$ is the surface tension, $\mathrm{N} / \mathrm{m}$, but the specific meaning of the surface tension $\sigma$ is not clearly stated, whether it is related to fluid viscosity is also not clear, and there is no evidence for the demonstration.

\subsection{Comparison with Taitel \& Dukler(1976) criterion}

The criterion relations (21) and (22) proposed in this study are derived from the basic model equation of stratified flow, and it is derived through strict mathematical derivation without referring any empirical relations. It is quite different from Taitel-Dukler criteria: the criterion of this study is derived by using the dynamic model, which considers the change of liquid level height with the change of time when the instantaneous flow rate of gas and liquid changes, and only two common assumptions in the study of two-phase flow are cited in the derivation.

Taitel \& Dukler(1976) proposed the transition condition expression (1) of $K_{T D}=1-h_{L} / d$ from stratified flow to non-stratified flow by extending the Kelvin-Helmholtz stability theory. The expression of $K_{T D}$ is a function of liquid level height and pipe diameter, and the relation of its function has no theoretical basis. In the criterion formula proposed in this study, $K_{V}$ is a function of density, liquid level height and wave velocity, while wave velocity $C_{V}$ is a function of viscosity, which is obtained through strict mathematical derivation. Therefore, this criterion synthesizes the influence of various influence factors on the stability of liquid level structure.

\subsection{The algorithm of flow pattern transition criterion for} stratified flow

When judging the flow pattern by using the criterion formula, firstly, it is necessary to use equations (10) and (11) to obtain the calculation formula of liquid level height $h_{L}$ under operating conditions. Therefore, the structural equation and geometric equation are substituted into equation (11), and the superficial velocity of gas and liquid phase was used to replace the real velocity of gas and liquid phase. The dimensionless number of pipe diameter $d$ and $d^{2}$ are determined by the liquid wetted perimeter $\mathrm{S}$ and cross-sectional area A respectively, and the final expression of $\mathrm{F}$ is obtained:

$$
\begin{aligned}
& F=\frac{f_{L} \rho_{L}\left(\frac{\pi}{4}\right)^{2} \tilde{S}_{L} w_{S L}^{2}}{2 d \tilde{A}_{L}^{3}}-\frac{f_{G} \rho_{G}\left(\frac{\pi}{4}\right)^{2} \tilde{S}_{G} w_{S G}^{2}}{2 d \tilde{A}_{G}^{3}} \mp \\
& \frac{f_{I} \rho_{G}\left(\frac{\pi}{4}\right)^{3} \tilde{S}_{I}}{2 d}\left[\frac{w_{S G}^{2}}{\tilde{A}_{L} \tilde{A}_{G}^{3}}-\frac{2 w_{S L} w_{S G}}{\tilde{A}_{L}^{2} \tilde{A}_{G}^{2}}+\frac{w_{S L}^{2}}{\tilde{A}_{G} \tilde{A}_{L}^{3}}+\left(\rho_{L}-\rho_{G}\right) g \sin \right]
\end{aligned}
$$

In the above formula, when $w_{G}>w_{L}$, the third term on the right side of the equal sign takes the minus sign, otherwise, takes the plus sign. For the steady state flow. $F=0$. If any two parameters of $w_{S L}, w_{S G}, h_{L}$ are known, the third unknown parameter can be solved from the above equation by using the iterative method. Since the above equation is a complex implicit equation about liquid level height, its solution requires numerical iterative calculation. Moreover, since $h_{L} / d$ should be strictly limited between 0 and 1 , otherwise, it will be difficult to obtain its stable numerical solution. At the same time, there may be 1 3 real roots in this equation, therefore, the binary method combined with the parabola method are chosen to calculate because of its good stability and all the real roots can be obtained by this method. And the partial derivatives of parameter $C_{V}$ are also obtained from the above formula by using numerical differentiation, that is, the extrapolated numerical differentiation method with fourth-order accuracy is used for calculation.

The calculation steps for judging the existence of stratified flow by using criterion formula (21) are as follows: (1)Giving a superficial velocity $w_{S G}$ of gas phase; (2)Supposing some dimensionless liquid level height value $h_{L} / d$ (For example, let $h_{L} / d=0.5$ ) ; (3) According to equation (25), $w_{S L}$ at this time and the partial derivative of $\mathrm{F}$ with respect to superficial velocity of gas-liquid phase can be solved, and $K_{V}$ can be obtained; (4)Substituting it into the criterion formula (20), and judge whether the equation is true. If it is true, the $h_{L} / d$ set and $w_{S L}$ solved both meet the requirement; (5)If it is not true, setting $h_{L} / d$ again, and return to step (3) to recalculate, until the equation is true.

\section{ANALYSIS ON THE INFLUENCE CRITERION FROM STRATIFIED FLOW}

\subsection{The influence of fluid properties on the transition criterion of stratified flow}

Based on the above algorithm, using large-scale horizontal DN80 experimental loop (Details of devices v processes and data collection points could be seen in references the research of Xiao et al), for liquid phases with different viscosity (Such as water、kerosene and lube base oil HVIW150), data collection and calculation are carried out respectively when the flow is stratified in horizontal pipe, the corresponding critical liquid phase superficial velocity values at different gas phase superficial velocities can be obtained. Plotting these values in a double logarithmic coordinate to obtain the relationship curve between them, the stable existence area of stratified flow is below 
the curve, the existence area of slug flow is above the curve, and the existence area of annular flow is on the right side of the curve. For Taitel \& Dukler criterion (1) and D.Barnea \& Y. Taitel criterion (24), the critical relationship curve of liquid level stability under the corresponding criterion can also be obtained by using the same calculation method, as shown in Figure 3.
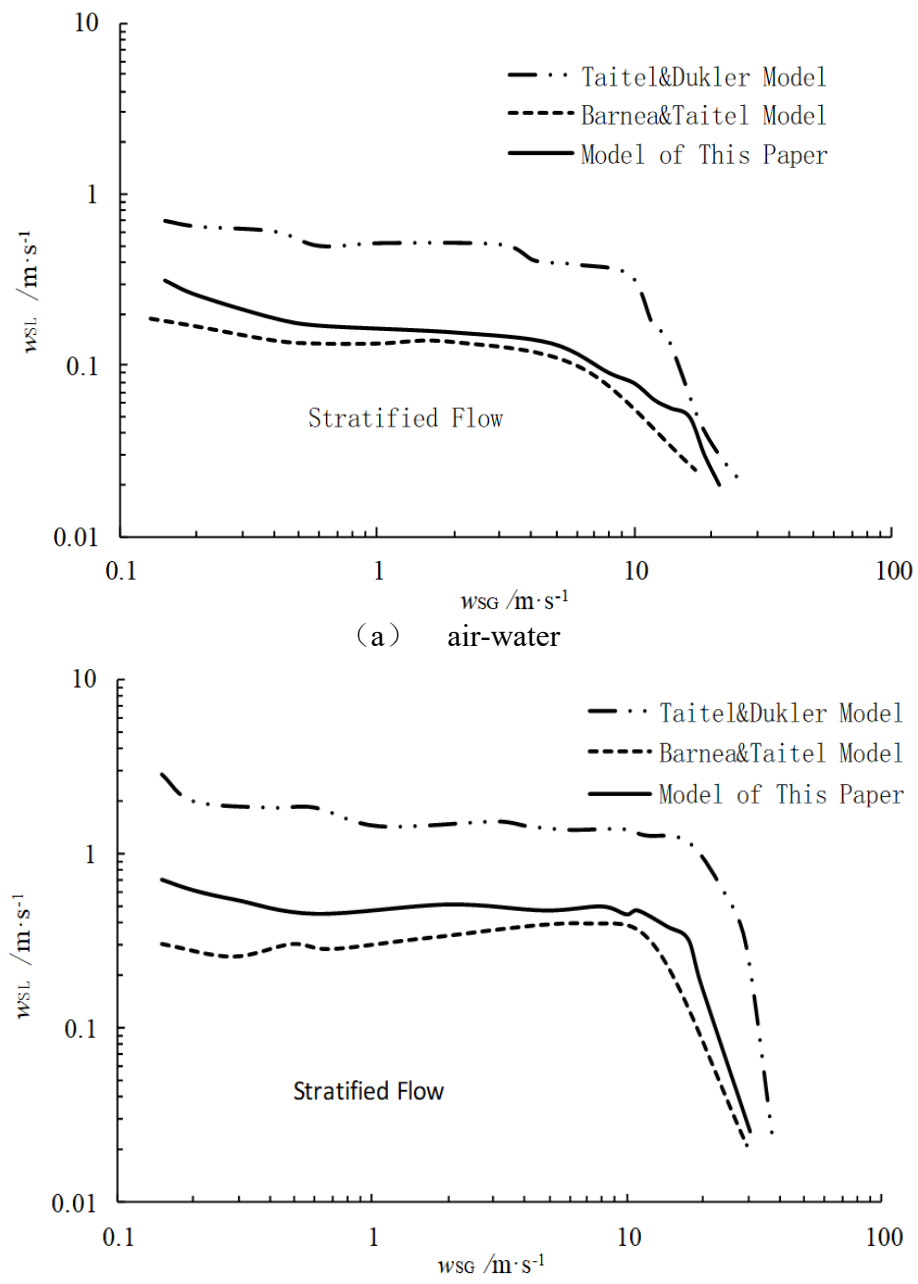

(b) air-kerosene

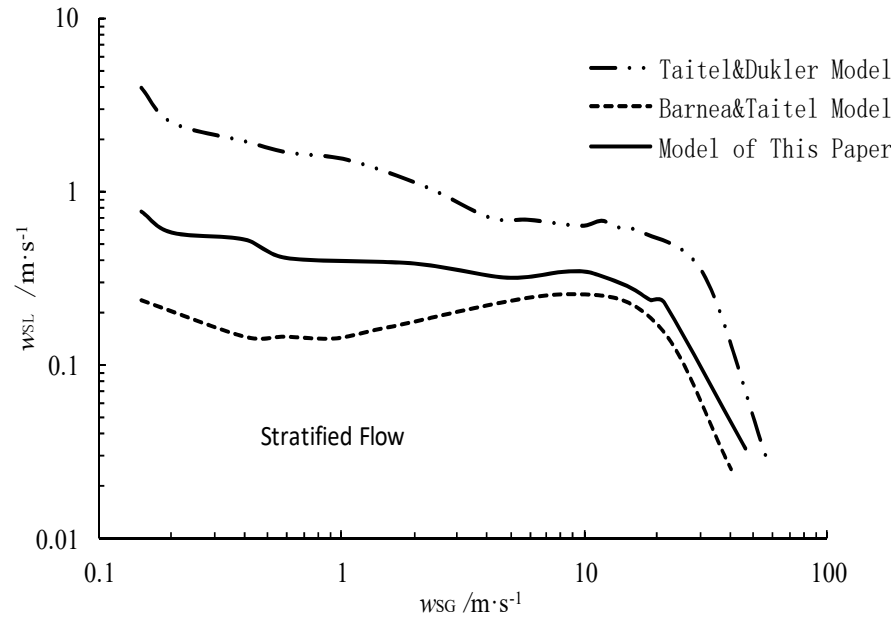

(c) air-lube base oil HVIW150

Fig. 3 Critical curve of stratified flow in horizontal pipe

For the horizontal gas-liquid two-phase flow pipeline, it can be seen from Figure 3 that when water is used as the liquid phase,
D.Barnea \& Y. Taitel (1993) is in good agreement with the predicted results of the criterion relationship in this study, and the stable existence area of stratified flow is basically consistent; when the oil with higher viscosity is used as the liquid phase, D.Barnea \&Y. Taitel(1993) and the calculation result of the criterion formula in this study have a large deviation when the gas phase superficial velocity is smaller. The deviation decreases gradually with the increase of gas phase superficial velocity; whether the liquid medium is water or oil, the Taitel \& Dukler criterion has a large deviation. The above research shows that liquid viscosity has a great influence on the stability of stratified flow. With the increase of liquid viscosity, the friction resistance and viscous force increase, and they play a role in preventing the rise of liquid level height, it is difficult to form liquid slug in the pipe, so that the region where the stratified flow exists stably is enlarged.

\subsection{The influence of instantaneous flow rate change of gas- liquid phase on the transition criterion of stratified flow}

In view of the gas-liquid two-phase flow in horizontal pipeline and wellbore, Dr. Ouyang from Stanford university proposed the transition condition of gas-liquid two-phase flow from stratified flow to nonstratified flow in wellbore on the basis of the transition theory from stratified flow to non-stratified flow proposed by Taitel \& Dukler (1976), which considered the influence of instantaneous flow rate change on flow pattern transition condition:

$w_{G}>\left(1-\frac{h_{L}}{D}\right)\left\{\frac{A_{G}}{\frac{d A_{L}}{d h_{L}}}\left[\frac{\rho_{L} g \cos \theta}{\rho_{G}}+\frac{V_{\mathrm{Im}} \mid V_{\mathrm{Im}}}{2 h_{G}}\right]\right\}^{0.5}$

The transition criterion expression from stratified flow to nonstratified flow proposed by Dr. Ouyang from Stanford university was based on Taitel \& Dukler(1976) theory, which considered the instantaneous flow rate change of gas and liquid. Compared with the traditional Taitel \&Dukler model, this formula increases the influence term of instantaneous gas-liquid flow rate change. When the instantaneous gas-liquid flow rate changed to 0 , then the formula will convert to Taitel \&Dukler formula. This formula is not only the discriminant of the transition from stratified flow to non-stratified flow, but also the condition that the wave increases at the gas-liquid interface when the liquid level in the pipe is high and the wave peak is close to the top of the pipe.

Its specific physical significance is as follows: when the gas-phase flow velocity at the gas-liquid smooth interface is greater than a specific value, the pressure difference caused by the change of the gas-phase flow velocity will be greater than the potential energy of the wave in the airflow, making the wave of the wavy flow increase. At this time, if the liquid level in the pipe is high and the wave peak is close to the top of the pipe, the wave peak closed to the top of the pipe will continue to rise and touch the wall at the top of the pipe. As a result, the stratified flow is converted into non-stratified flow (slug flow or annular fog flow (That is, annular flow with droplets entrainment)).

The traditional model fails to take into account the influence of instantaneous flow rate change of each phase on flow pattern transition condition. However, when the instantaneous flow rate changes, the friction coefficient between the gas-liquid phase and the pipe wall and the friction coefficient between the gas-liquid phase will also change. The friction coefficient is the main parameter that causes the closure of the transient state flow equation of gas-liquid two-phase flow. The change of the friction coefficient will correspondingly change the value of the liquid holdup, which will eventually lead to the flow pattern transition. So the traditional flow pattern transition model cannot be applied to predict the flow pattern transition from stratified flow to nonstratified flow under the condition that flow rate of each phase change instantaneously. Based on the D.Barnea \&Y. Taitel(1993) model, the format of influence term of instantaneous flow rate change of gas and liquid proposed by Dr. Ouyang is used in this study, and the transition condition of gas-liquid two-phase flow from stratified flow to nonstratified is also obtained: 


$$
w_{G}>K_{V}\left\{\frac{A_{G}}{\frac{d A_{L}}{d h_{L}}}\left[\frac{\rho_{L} g \cos \theta}{\rho_{G}}+\frac{V_{\mathrm{Im}}\left|V_{\mathrm{Im}}\right|}{2 h_{G}}\right]\right\}^{0.5}
$$

In equation (27), $V_{\mathrm{Im}}$ is the volume flow rate of inflow, $\mathrm{m} 3 / \mathrm{s}$; $h_{G}$ is the height of the gas phase in the pipe, m; The expression of $K_{V}$ is the same as the transition model from stratified flow to non-stratified flow proposed by D.Barnea \& Y. Taitel, but since it increases the influence term $\frac{V_{\text {Im }}\left|V_{\text {Im }}\right|}{2 h_{G}}$ of the instantaneous flow rate change of gasliquid flow, it is essentially the transition criterion equation (22) from stratified flow to non-stratified flow proposed in this study which considered the instantaneous change of gas-liquid flow rate.



Figure. 4. Observation results of gas-liquid two-phase flow pattern at experimental points

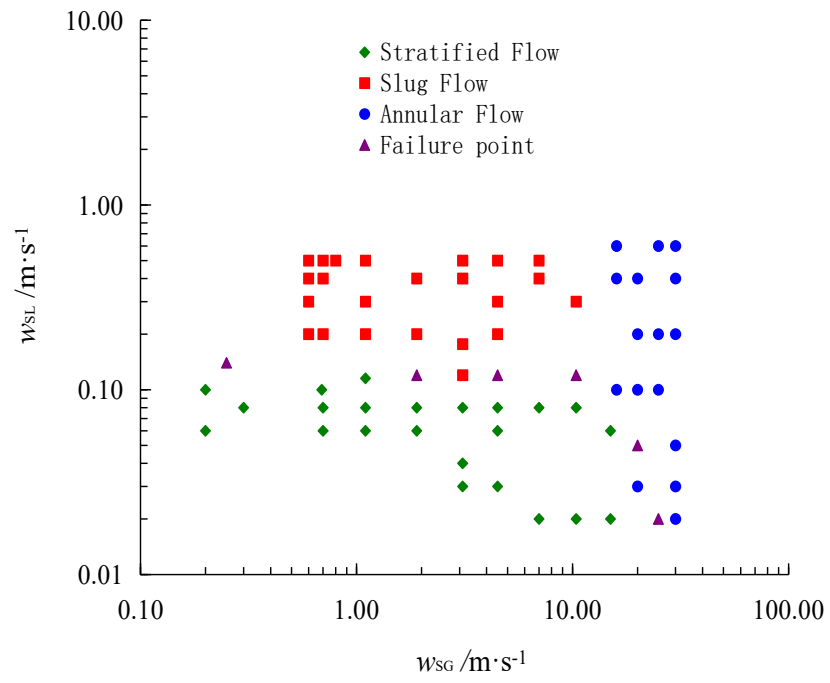

Figure. 5 Predicted results of the comprehensive flow pattern criterion at experimental points of gas-liquid two-phase flow

The experimental study on the flow pattern transition of instantaneous flow rate change of gas-liquid phase is conducted on the loop used in this study. The experimental medium is air-water, and setting that flow rate of the liquid water changes along the radial direction in the experimental pipe section. When the flow rate of gasliquid two-phase flow changes instantaneously, the flow pattern at the experimental point of gas-liquid two-phase flow observed in the experiment is shown in Figure 4. Equation (27) is used to predict the flow pattern of all gas-liquid two-phase experimental points with instantaneous change of gas-liquid flow rate, and the results are shown in figure 5 .

According to the comparative analysis in figure 4 and figure 5 , it can be concluded that the comprehensive prediction criterion of flow pattern transition from stratified flow to non-stratified flow proposed in this study is adopted to predict the flow pattern of all experimental points, and the non-conformity rate is $8.33 \%$, that is, $6 / 72$, which can fully meet the requirements of engineering accuracy.

The prediction effects of three different flow pattern prediction models are analyzed. In the multiphase pipeline experiment in this study, the instantaneous change of gas-liquid flow rate per unit length is $0.003 \mathrm{~m}^{2} / \mathrm{s}$. This experiment could be used to illustrate the flow pattern transition in the actual horizontal pipe. See tables 1 and 2.

As can be seen from table 1 and table 2, in regard to the prediction of the flow pattern transition of gas-liquid two-phase from stratified flow to non-stratified flow, the prediction effect on flow pattern of the model proposed in this study based on the Dr. Ouyang and D.Barnea model is relatively good, but the prediction failure area is mainly located in the region with low liquid phase superficial velocity and high gas phase superficial velocity. Three flow pattern transition prediction models are used to predict the flow pattern under the four working conditions in which the flow rate change is $0 \mathrm{~m}^{2} / \mathrm{s}, 0.003 \mathrm{~m}^{2} / \mathrm{s}, 0.006 \mathrm{~m}^{2} / \mathrm{s}$ and $0.009 \mathrm{~m}^{2} / \mathrm{s}$, according to the flow pattern prediction results, the flow pattern changed at only 4 experimental points (located in the transition area of flow pattern transition) among 72 experimental points. The influence term of instantaneous change of gas-liquid flow rate has little influence on the flow pattern transition of local units, therefore, the influence term of instantaneous change of gas-liquid flow rate can be ignored. In the actual pipeline, the transition of flow pattern may occur when the flow rate of gas-liquid two-phase flow changes instantaneously. However, in the actual gas condensate pipes and wet gas wells, due to the flow rate of gas-liquid two-phase flow varies constantly with the temperature and pressure, the instantaneous change of fluid phase state has a great influence on the change of superficial velocity of each phase of downstream pipeline unit, so its influence on the flow pattern of downstream pipeline unit cannot be ignored, the prediction of flow pattern in wet natural gas horizontal wellbore should be carried out in sections.

\section{Conclusion}

(1) Aiming at the flow pattern transition between stratified flow and non-stratified flow, based on the Viscous Kelvin-Helmholtz model proposed by D.Barnea \& Y.Taitel (1993), the transition criterion from stratified flow to non-stratified flow is obtained. Compared with the criteria of D Barnea \& Y. Taitel (1993) and Taitel \& Dukler(1976), the algorithm of flow pattern transition criterion of stratified flow and the calculation steps for judging the existence of stratified flow is given; (2) On the basis of theoretical mechanism study, using the experimental data of large loop multiphase flow, the influence of liquid viscosity and the instantaneous volume flow rate change of gas-liquid two-phase on the transition criterion is analyzed. And the adaptability of various flow pattern transition criteria which used to predict the flow pattern at the experimental point is evaluated. The result shows that liquid viscosity has a great influence on the stability of stratified flow. With the increase of liquid viscosity, the friction resistance and viscous force increase, and they play a role in preventing the rise of liquid level height, it is difficult to form liquid slug in the pipe, so that the region where the stratified flow exists stably is enlarged. And instantaneous change of gas-liquid flow rate has little influence on the flow pattern transition. 
Table. 1. The influence of gas-liquid two-phase flow rate instantaneous changes on the predicted results of the three different flow pattern prediction models

\begin{tabular}{|c|c|c|c|c|}
\hline & & \multicolumn{3}{|c|}{$\begin{array}{l}\text { Compared with no instantaneous change of gas-liquid flow rate, the influence of the } \\
\text { instantaneous change of different gas-liquid flow rate }\end{array}$} \\
\hline \multicolumn{2}{|c|}{ The instantaneous change of gas-liquid flow rate $q_{I}, \mathrm{~m}^{2} / \mathrm{s}$} & 0.003 & 0.006 & 0.009 \\
\hline \multicolumn{2}{|c|}{ Experimental observation } & $4 / 72$ & l & l \\
\hline \multirow{3}{*}{$\begin{array}{l}\text { Prediction model of flow pattern } \\
\text { transition }\end{array}$} & Taitel \& Dukler & $0 / 65$ & $0 / 65$ & $0 / 65$ \\
\hline & D.Barnea \&Y. Taitel & $18 / 72$ & $23 / 72$ & $23 / 72$ \\
\hline & $\begin{array}{l}\text { Research model of this } \\
\text { paper }\end{array}$ & $6 / 72$ & $8 / 72$ & $8 / 72$ \\
\hline
\end{tabular}

Table. 2. Prediction coincidence rate of three different flow pattern prediction models when the volume flow rate change of gas-liquid two-phase is $0.003 \mathrm{~m}^{2} / \mathrm{s}$

\begin{tabular}{|c|c|c|c|c|}
\hline Flow pattern transition criterion & Taitel \& Dukler & D.Barnea \&Y. Taitel & Ouyang & Research model of this paper \\
\hline Prediction coincidence rate & $27 / 72$ & $51 / 72$ & $57 / 72$ & $57 / 72$ \\
\hline
\end{tabular}

\section{ACKNOWLEDGE}

This work was supported by the Shaanxi Education Department Service Local Special Program Project (19JC034); Xi'an Petroleum University Youth Science and Technology Innovation Fund (NO. 2015BS011) and Xi'an Petroleum University postgraduate innovation and practice ability training program (YCS20213145), so grateful here.

\section{NOMENCLATURE}

$w_{G}, w_{L^{--}}$Real velocity of gas phase and liquid phase, $\mathrm{m} / \mathrm{s}$

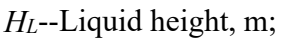

$q_{I G}, q_{I L}$--Volume flow rate of gas phase and liquid phase in unit length pipeline, $\mathrm{m}^{2} / \mathrm{s}$;

$\rho_{G}, \rho_{L}$-- Density of gas phase and liquid phase, $\mathrm{kg} / \mathrm{m}^{3}$;

$A_{G}, A_{L^{--}}$Cross sectional area of gas phase and liquid phase in pipeline, $\mathrm{m}^{2}$;

$\tau_{W G}, \tau_{W L}, \tau_{I}$-- Shear stress of gas- wall, liquid- wall and gas- liquid phase, $\mathrm{Pa}$;

$P_{I G}, P_{I L}$-- Pressure gradient of gas phase and liquid phase, $\mathrm{Pa} / \mathrm{m}$;

$S_{L}, S_{G}, S_{I}$-- Liquid wet perimeter, gas wet perimeter and gas-liquid interface length, $\mathrm{m}$;

$\frac{\partial P}{\partial x}$-- Pressure gradient, $\mathrm{Pa} / \mathrm{m}$;

$\varphi_{\text {-- }}$ Void fraction of cross section;

$C_{V}$-- Propagation velocity of interface unstable wave;

$C_{I V}$-- Inviscid critical interface wave velocity;

$v_{L}$-- Kinematic viscosity of liquid;

S-- Barrier coefficient;

$\sigma$--Surface tension, $\mathrm{N} / \mathrm{m}$;

\section{REFERENCES}

Spedding P.L., Spence D.R. Flow regimes in two-phase gas-liquid flow [J]. Int. J. Multiphase Flow, 1993, 9(2): 245-280.

https://doi.org/10.1016/0301-9322(93)90002-C

Espedal M. An Experimental investigation of stratified two-phase pipe flow at small inclinations [D]. Department of mechanical engineering, NTNU, Norway, 1998.

https://doi.org/10.1016/j.ijmultiphaseflow.2010.03.002
Meng, W. Low-Liquid Loading Gas-Liquid Two-Phase Flow in Near Horizontal Pipes [D]. The University of Tulsa, Tulsa, Oklahoma, 1999. https://doi.org/10.2118/74687-PA

Nicolas R, Zhang H, Wang Q, REDUS, L Clifford, BRILL, P James. Experimental Study of Low-Liquid-Loading Gas-Liquid Flow in NearHorizontal Pipes [J]. Spe Production \& Facilities, 2001, 16(4):240-249. https://doi.org/10.2118/74687-PA

Badie S, Hale C P, Lawrence C J, Hewitt G F. Pressure Gradient and Holdup in Horizontal two-phase gas-liquid flows with Low Liquid Loading [J]. Int. J. Multiphase Flow, 2000, 26(9): 1525-1543.

https://doi.org/10.1016/S0301-9322(99)00102-0

Barnea D, Taitel Y. Kelvin-Helmholtz stability criteria for stratified flow: viscous versus non-viscous (inviscid) approaches [J]. Int. J. Multiphase Flow. 1993, 19(4): 639-649.

https://doi.org/10.1016/0301-9322(93)90092-9

Oliemans, R.V.A. Multiphase science and technology for oil/gas production and transport [C]. The University of Tulsa Centennial Petroleum Symposium, Tusa, O.K., U.S.A., Aug. 29-31, 1995: 9-32. https://doi.org/10.2118/27958-MS

Beggs.H.D, Brill.J.P. A study of two-phase flow in inclined pipes [J]. J.Pet.Technol, May, 1973: 607-617. https://doi.org/10.2118/4007-PA

Taitel.Y, Dukler.A. E., A model for predicting flow regime transitions in horizontal and near horizontal gas-liquid flow [J]. AICHE, 1976, 22, No.1: 47-55.

https://doi.org/10.1002/aic.690220105

Dukler A.E., Taitel.Y. Flow pattern transitions in gas/liquid system [J]. Int Hewitt. Mutiphase Science and Technology. Washington, 1986, 2: 148-161.

https://doi.org/10.1615/MultScienTechn.v2.i1-4.10

Taitel Y, Bornea D, Dukler A E. Modelling flow pattern transitions for steady upward gas-liquid flow in vertical tubes [J]. AIChE Journal, 1980, 26(3):345-354. 


\section{https://doi.org/10.1002/aic.690260304}

Venkateswararao P, Semiat R, Dukler A E. Flow pattern transition for gas-liquid flow in a vertical rod bundle [J]. International Journal of Multiphase Flow, 1982, 8(5):509-524.

https://doi.org/10.1016/0301-9322(82)90021-0

Krishnan V S, Kowalski J E. Study of the stratified-slug flow transition in a horizontal pipe containing a rod bundle [J]. Annual Symposium on Simulation of Reactor Dynamics \& Plant Control, 1985, 10. https://doi.org/10.1016/0029-5493(87)90136-1

L.E.Gomez, Ovadia Shoham, Zelimir Schmidt, R. N. Chokshi, Tor Northug. Unified Mechanistic Model for Steady-State Two-Phase Flow: Horizontal to Vertical Upward Flow [J]. SPE Journal, 2000,5(3):339350.

https://doi.org/10.2118/65705-PA

Yuri V. Fairuzov. Stability analysis of stratified oil/water flow in inclined pipelines [J]. SPE Production and Facilities, 2001, 16(1):14-21. https://doi.org/10.2118/58934-PA

Jingyu X. The Effects of Liquid Properties on Flow Pattern Transition and Pressure Drop During Gas-liquid Pipe Flow [J]. Journal of Basic Science \& Engineering, 2005, 13(2):111-119.

https://doi.org/10.1360/biodiv.050084

Hu Z H, Yang Y, Liu L, Zhou F. Local Flow Regime Transition Criteria of Gas-Liquid Two-phase Flow in Vertical Upward Tube with a Horizontal Rod [J]. Chinese Journal of Chemical Engineering, 2006, 14(4):442-449.

https://doi.org/10.1016/S1004-9541(06)60097-9

Liu Y P, Yang W L, Wang J. Influence of gas-liquid interfacial friction factor on the transition from stratified to slug flow [J]. Journal of Chemical Engineering of Chinese Universities, 2006, 20(6):888-892.

https://doi.org/10.1016/j.mineng.2005.09.006

Liu Y P, Yang W L, Wang J. Experimental study for the stratified to slug flow regime transition mechanism of gas-oil two-phase flow in horizontal pipe [J]. Frontiers of Energy and Power Engineering in China, 2008, 2(2):152-157.

https://doi.org/10.1007/s11708-008-0012-7

Zhang J H, Yan C Q, Sun Z N. Investigation of flow pattern transition for gas-liquid two-phase flow in horizontal rolling pipes [J]. Journal of Harbin Engineering University, 2008, 29(10): 1050-1053.

https://doi.org/10.1088/0256-307X/25/2/069

Wang X, Sun X, Doup B, Zhao H. Dynamic Modeling Strategy for Flow Regime Transition in Gas-Liquid Two-Phase Flows [J]. Journal of Computational Multiphase Flows, 2011, 4(4):387-398.

https://doi.org/10.1260/1757-482X.4.4.387

De F L, Liu R, Lu Q H. The Flow Pattern Transition Identification and Interphases Force Detection of Gas-Liquid Two-Phase Flow [J]. AASRI Procedia, 2012, 3(Complete):534-539.

https://doi.org/10.1016/j.aasri.2012.11.084
Xiao R, Wang Y, Pan J, Wei B, Chen G. Transition between stratified and non-stratified flow pattern for gas-liquid flow in horizontal porous pipe [J]. Ciesc Journal, 2013, 64(10):3606-3611. https://doi.org/10.3969/j.issn.0438-1157.2013.10.016

Bhagwat S M, Ghajar A J. An Empirical Model to Predict the Transition Between Stratified and Non-stratified Gas-Liquid TwoPhase Flow in Horizontal and Downward Inclined Pipes [J]. Heat Transfer Engineering, 2015, 36(18):1485-1494. https://doi.org/10.1080/01457632.2015.1024979

Eissa Al-Safran, Khaled Al-Qenae. A Study of Flow-Pattern Transitions in High-Viscosity Oil-and-Gas Two-Phase Flow in Horizontal Pipes [J]. SPE Production \& Operations, 2018, 33(2):269280.

https://doi.org/10.2118/187939-PA

Fedor Ronshin, Evgeny Chinnov. Experimental characterization of twophase flow patterns in a slit microchannel [J]. Experimental Thermal and Fluid Science, 2019, 103:262-273.

https://doi.org/10.1016/j.expthermflusci.2019.01.022

Weisman J. Effects of fluid properties and pipe diameter on two phase flow patterns in horizontal lines [J]. Int. J. Multiphase Flow, 1979, 5: 437-462.

https://doi.org/10.1016/0301-9322(79)90031-4

Hongkun Dong. An Experimental Study of Low Liquid Loading GasOil-Water Flow in Horizontal Pipes [D]. The university of TULSA, 2007.

Xiao R G, Dong Z Y. An Experimental Investigation on Pigging Transient Characteristics of Gas-Liquid Two-phase Flow in Horizontal Pipes [J], Journal of Oil \& Gas Storage and Transportation, 2007, 26(4) : 21-25.

https://doi.org/10.3969/j.issn.1000-8241-D.2007.04.007

Xiao R G, Wang L Y, Deng Z A, Guo X A, Zhang L. Evaluation of the formulas for calculating the interfacial friction factor of the two-phase stratified flow in horizontal condensate natural gas pipeline [J], Journal of Xi'an Shiyou University (Naturnal Science Edition), 2006, 21(3): 5557.

https://doi.org/10.3969/j.issn.1673-064X.2006.03.015

Ouyang L B, Aziz K. A simplified approach to couple wellbore flow and reservoir inflow for arbitrary well configurations [J]. SPE Annual Technical Conference and Exhibition, New Orleans, Louisiana. September, 1998: 27-30. https://doi.org/10.2118/48936-MS

Wu S Q, Zhong X F, Liu X B, Pei C X. Tech of Production Profile Logging in Horizontal Well and Its Application [J]. Well Testing, 2005, 14(2): 57-59.

https://doi.org/10.3969/j.issn.1004-4388.2005.02.021

Li X P, Zhang L H, Li Y, Chen H L. Variation laws of pressure and production rate in horizontal well [J], Hydrodynamic research and progress series A, 2005, 20(4): 492-496.

https://doi.org/10.3969/j.issn.1000-4874.2005.04.013 